\title{
Efficient Fuzzy Logic-Based Clustering Algorithm for Wireless Sensor Networks
}

\author{
Moazam Bidaki ${ }^{1}$, Seyed Reza Kamel Tabbakh ${ }^{2}$ \\ ${ }^{1}$ Department of Computer Engineering, Neyshabur Branch, Islamic Azad \\ University, Neyshabur, Iran, \\ Bidaki@Iau-neyshabur.ac.ir \\ 2 Department of Software Engineering, Mashhad Branch,Islamic Azad University, \\ Mashhad, Iran, \\ rezakamel@computer.org
}

\begin{abstract}
Clustering is one of the main techniques used to increase the scalability of wireless sensor networks (WSNs). Furthermore, clustering can help to improve the energy efficiency of resource limited ad hoc network and increase the lifetime of sensor network. In this paper, we present a fuzzy clustering algorithm which improves the energy efficiency of LEACH protocol by using a better cluster head selection solution. For this propose, we apply the number of live neighboring nodes and remaining energy of network nodes to select better nodes for clustering. By creating more symmetric clusters, our scheme reduces the energy consumption of the sensors and this finally increases the lifetime of the sensor network. Finally, we simulate our solution in MATLAB software and compare it with the LEACH clustering protocol in various scenarios.
\end{abstract}

Keywords: Energy, Clustering, Fuzzy Logic, LEACH

\section{Introduction}

A wireless sensor network (WSN) consists of a large number of miniaturized sensor nodes which collect data form environment and send them to the base station via radio transmitter[1]. To keep the cost and size of these sensors small, they are equipped with small batteries, which restrict them to have limited power and computational capacity. This puts significant constraints on the power available for communications, and affects the data rate as well as transmission range[2]. However, against such limitations, WSNs can be used in various applications such as military, biomedical, and environmental applications[3].

Clustering provides an efficient way to save energy inside WSNs and enables efficient resource allocation and it also improves the WSN's scalability.

Generally, clustering in WSNs is defined as the process of dividing the network nodes into groups, where each group agrees on a central node, called the cluster head[4].

The cluster head or $\mathrm{CH}$ acts as a bridge between other sensor nodes and base station and sometimes between one cluster head and other cluster head in multi-hop cases[5]. Cluster heads have responsibility of locality preserving, adding or leaving of nodes, scheduling time slots for each node, data aggregation and management of messages between nodes and base station[4, 6, 7]. Sensors deployed in different regions collaborate to gather data and sends to cluster head which is further transferred to base station or another cluster head after processing LEACH is one of the established clustering based routing protocols in WSN[8]. The selection of cluster head in LEACH is done randomly and the data that transmit between the cluster head and the base station is done directly which tend to exhaust the sensor battery quickly $[9,10]$. 


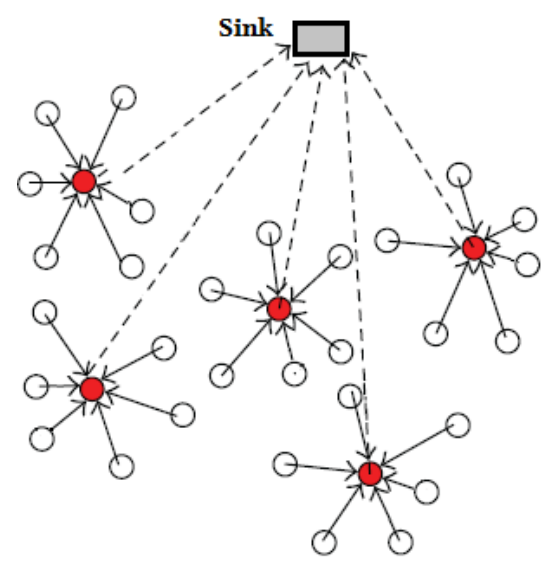

Figure 1. Network Clustering

In this paper, we propose a new fuzzy logic-based clustering algorithm which provides a more appropriate cluster head selection method by using the number of live neighboring nodes and remaining energy of network nodes. Our solution is based on the LEACH protocol which provides a probabilistic cluster head selection of random sensor nodes. This scheme improves the cluster head selection process and creates more symmetric clusters which results in fewer energy consumption and increase lifetime of WSN.

The rest of this paper is organized as follows. Section II describes the related fuzzy clustering solutions, section III explains the proposed clustering algorithm with details of this experiment. Section IV describes the parameters and simulation result

\section{Problem Statement}

LEACH or Low Energy Adaptive Clustering Hierarchy is proposed by Heinzelman et al. in [8]. In this protocol, time is divided into consecutive rounds and each round is consisting of set-up phase and steady state phase.

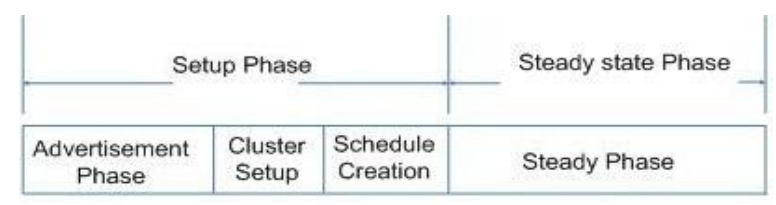

Figure 2. Clustering with LEACH

In set-up phase, cluster heads are elected and clusters are formed. In steady state phase, environmental data are sensed and transmitted to the cluster head. After a cluster head receives its members' data, it aggregates them and sends it to the sink. At the start of each set-up phase, each sensor node generates a random number between 0 and 1 to determine it will become a cluster-head or not, then compares this number with the threshold value $\mathrm{T}(\mathrm{n})$ which is computed by the following equation:

$$
T(n)= \begin{cases}\frac{P}{1-P *\left(\operatorname{rmod} \frac{1}{P}\right)} & \text { if } \mathrm{n} \in \mathrm{G} \\ 0 & \text { otherwise }\end{cases}
$$

Where $\mathrm{P}$ is the desired percentage of cluster heads, $\mathrm{r}=$ current round, and $\mathrm{G}$ is the set of nodes that have not been cluster-heads in the last $1 / \mathrm{P}$ rounds. If the number chosen by 
each node is less than the threshold value $\mathrm{T}(\mathrm{n})$, the node becomes a $\mathrm{CH}$ for that current round[11].

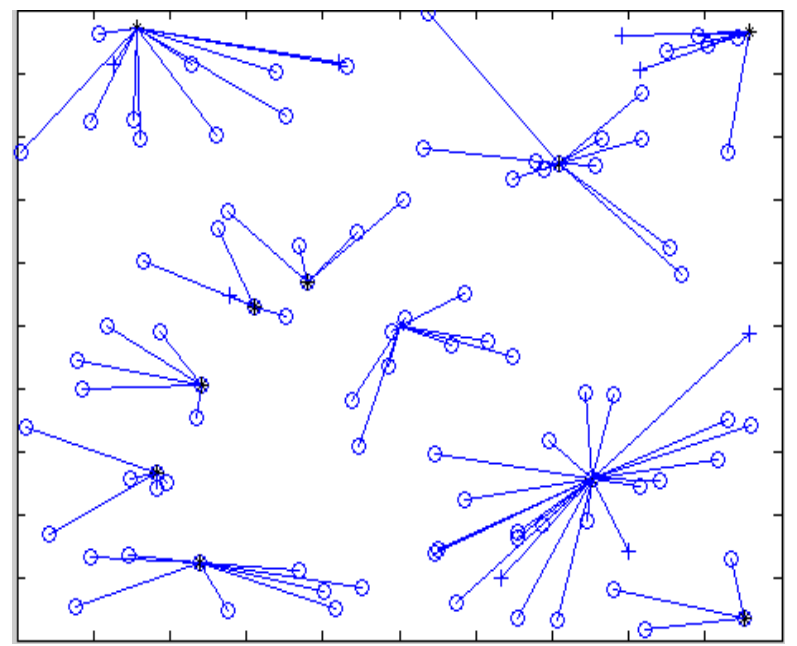

Figure 2. Clustering with LEACH

Although, LEACH is a reliable clustering and routing protocol in WSNs, it applies a probabilistic cluster head selection method which as indicated in Figure 2, it may cause the following problem:

- Because each node independently selects itself as cluster heads, the cluster heads may be positioned near to each other.

- Some nodes which have fewer neighboring nodes may be elected as cluster heads and network may not be utilized form advantages of data aggregation.

- Random selection of cluster heads does not create symmetric clusters and this may increase the total distance of nodes to cluster heads communications. As the distance of communications is increased more energy should be consumed to transfer the sensed data to cluster heads which this finally reduces the network lifetime.

\section{Related Works}

Many clustering schemes Fuzzy logic has been applied in in WSNs. For example, in [12] , a multihop clustering scheme is proposed which is called fuzzy clustering algorithm (FCA) which is aimed to prolong the lifetime of WSNs. The FCA adjusts the cluster-head radius considering the residual energy and the distance to the base station parameters of the sensor nodes. This helps decreasing the intra-cluster work of the sensor nodes which are closer to the base station or have lower battery level. They utilize fuzzy logic for handling the uncertainties in cluster-head radius estimation.

In [13] Kumar et al. propose an energy efficient hierarchical clustering scheme which uses the fuzzy logic method for cluster formation and cluster head selection. To determine the cluster head selection, three input functions convert the inputs into fuzzy sets, such as distance, node density and battery level. Also, this scheme applies 27 rules for the fuzzy inference. In [14] a fuzzy logic based energy-aware dynamic clustering technique is presented by Alim et al. to increase the network lifetime in terms of LND. In this scheme, residual energy and node centrality are given to the fuzzy inference system and a node is selected as a cluster head according to the fuzzy cost (output). Also, in this scheme, the optimum number of clusters is formed in every round, which is almost impossible in LEACH (low-energy adaptive clustering hierarchy). Moreover, this protocol has less computational load and complexity. 
In [15] Barolli et al. propose a power reduction algorithm for sensor networks based on fuzzy logic and node movement. This scheme uses four input linguistic parameters which are remaining power of sensor, degree of number of neighbor nodes, distance from cluster centroid and sensor speed for cluster-head decision.

An improved version of HEED protocol is presented in [16] by Neamatollahi et al which uses fuzzy logic and a non-probabilistic approach for $\mathrm{CH}$ election. Moreover, it uses the node's remaining energy and two fuzzy descriptors to elect CHs. The fuzzy variables are the number of neighbors a node and node centrality which node centrality is calculated as the average of the squared distances of neighbor nodes from a particular node. Since, typically transmission energy is proportional to the squared distance, the lower value of the node centrality results in a lower amount of energy required by other nodes to send data to that node as a $\mathrm{CH}$. In this solution, the output of fuzzy logic is the fuzzy cost and by an increase of node degree and decrease of node centrality, the cost of a node being elected as a $\mathrm{CH}$ is decreases which mean that the node has a higher priority of being elected a $\mathrm{CH}$.

In [9] Wang et al. propose a fuzzy-based algorithm for controlling sensor speed in WSNs. They use 4 input linguistic parameters for evaluating cluster-head decision probability which is used as a fuzzy input to control sensor speed. By considering the moving speed of the sensor they are able to predict whether the node will leave or stay in the cluster. This scheme applies the following

- Distance from Cluster Centroid (DCC);

- Degree of Number of Neighbor Nodes (D3N);

- Remaining Battery Power of Sensor (RPS);

- Sensor Speed (SS).

The authors in [17] introduce the idea of fuzzy logic according to the nodes' residual energy, communication range, computing power, neighbor nodes, etc. Using fuzzy comprehensive evaluation method in virtual cluster head selection and the formation of the wireless network hierarchy overcome the randomness of cluster head election in LEACH protocol and uneven distribution of node energy; at the same time, generating the cluster node consequence through network initialization reduces the energy consumption when network constantly selects the cluster head and prolongs the life cycle of wireless sensor network.

\section{Proposed Solution}

This section presents our proposed fuzzy-logic based clustering scheme. In this scheme, we modify the probabilistic cluster head selection process and present a nonprobabilistic approach which creates more symmetric clusters. As indicated in Figure 5, we assume a simple model for the radio hardware energy dissipation where the transmitter dissipates energy to run the radio electronics and the power amplifier, and the receiver dissipates energy to run the radio electronics. 


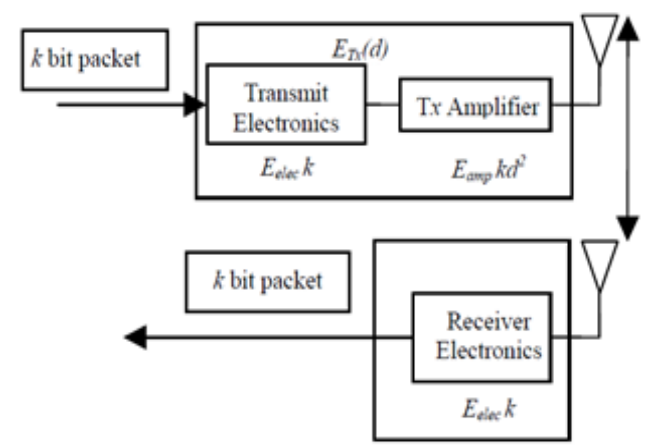

Figure 3. Radio Model

Thus, to transmit a $k$-bit message a distance $d$ using our radio model, the radio expends:

$E_{T X}(k, d)= \begin{cases}E_{\text {elec }} \times k+E_{f s} \times k \times d^{2} & \left(d<d_{0}\right) \\ E_{\text {elec }} \times k+E_{\text {mp }} \times k \times d^{4} & \left(d \geq d_{0}\right)\end{cases}$

In these equations $d_{0}$ can be computed as follows:

$d_{0}=\sqrt{E_{f s} / E_{m p}}$

Also, to receive this message, the radio expends:

$E_{R X}(k)=E_{\text {elec }} \times k$

As indicated in Figure 4, a fuzzy system basically consists of fuzzification, inference engine, and defuzzification parts. The fuzzifier maps each crisp input value to the corresponding fuzzy sets and assigns it a truth value or degree of membership for each fuzzy set. The fuzzified values are processed by the inference engine, which consists of a rule base and various methods for inferring the rules. The rule base is simply a series of IF-THEN rules that relate the input fuzzy variables with the output fuzzy variables using linguistic variables, each of which is described by a fuzzy set, and fuzzy implication operators AND, OR, etc.

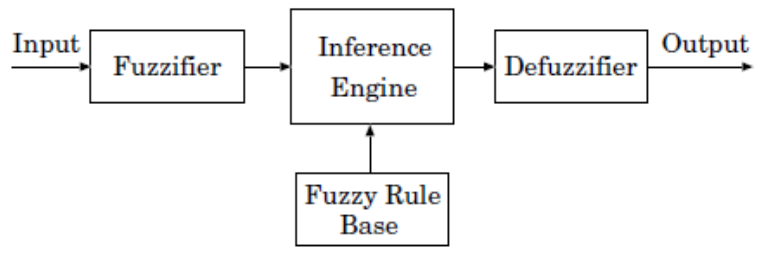

Figure 4. FIS Structure

The fuzzy system used in the inference engine of this scheme is the Mamdani fuzzy system which is a simple rule-base method that does not require complicated calculations and can employ the rules to control systems. The defuzzifier performs defuzzification on the fuzzy solution space and finds a single crisp output value from the solution fuzzy space. The fuzzy system inputs are crisp numbers that are converted to the fuzzy values by membership functions. The nodes simply determine these input values. The nodes are aware of Neighboring nodes and their distance, as soon as one sending and receiving data. 


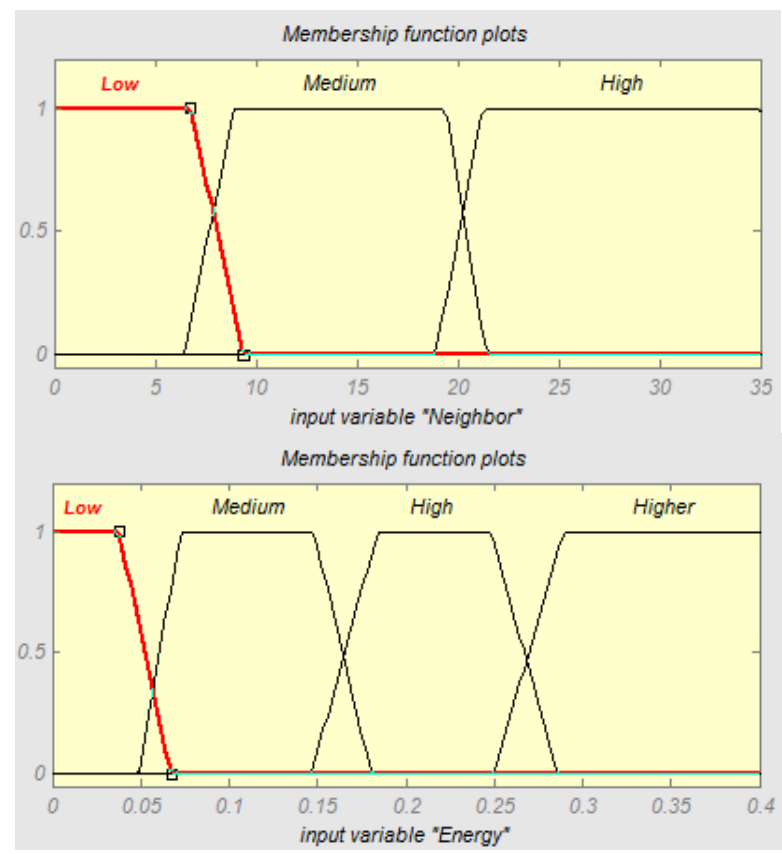

Figure 5. Membership Functions

Membership functions that covert crisp input values to fuzzy values are shown by Figure 5. As indicated in this figure, the number of live neighboring nodes and the remaining energy of the nodes are applied for network clustering. In this scheme, mamdani method uses 12 rules which are indicated in table 1.

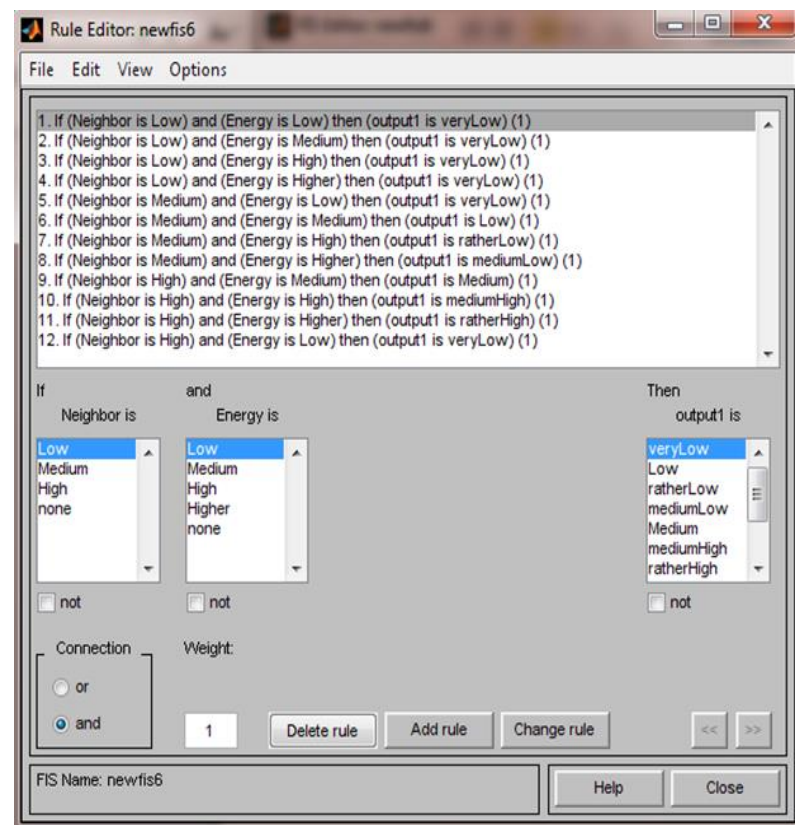

Figure 6.Fuzzy Rules

Figure 7 indicates the output membership function. In this paper, we use of Center of Area (COA) method to deffuzification output. 


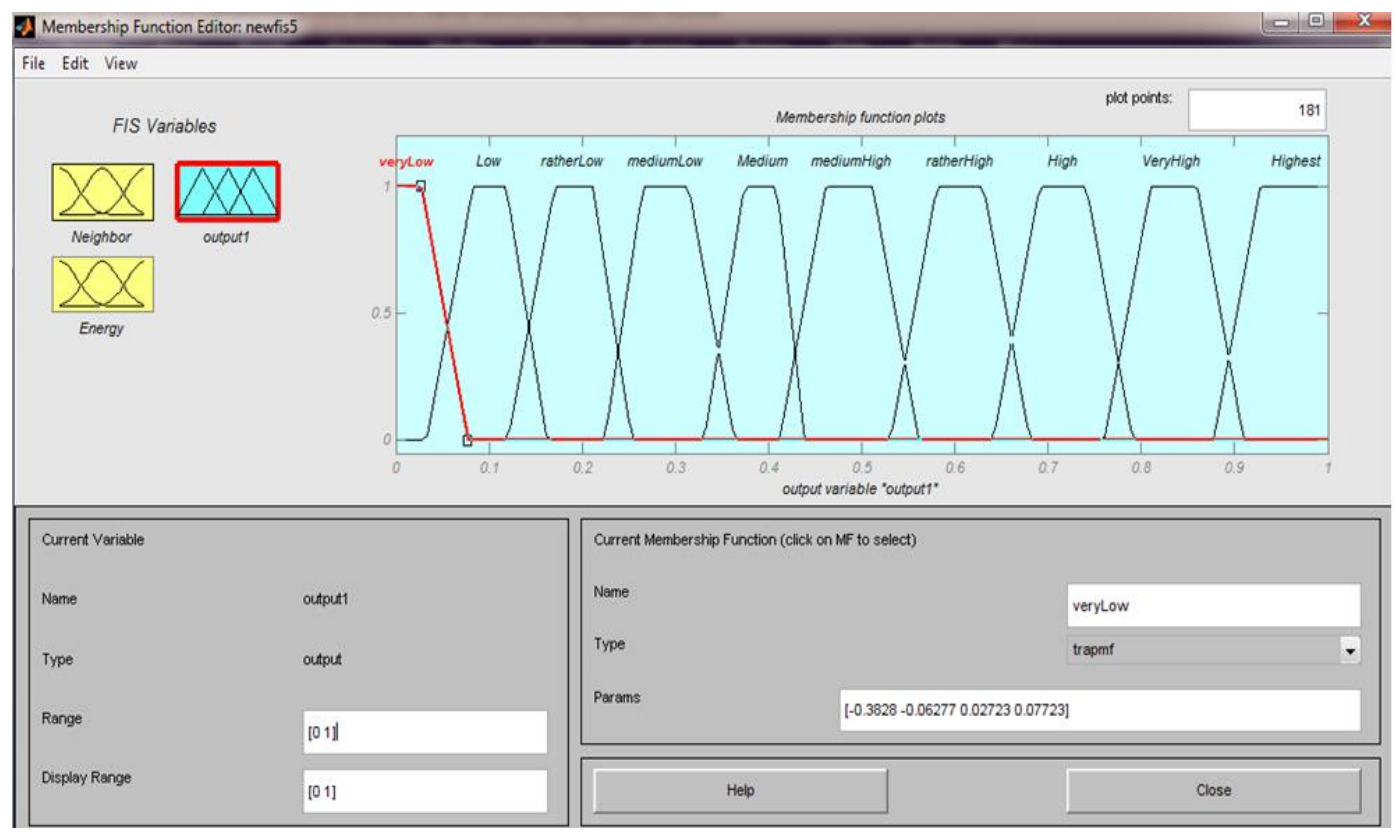

Figure 7. Output Membership Functions

\section{Simulation results}

This section presents the simulation results of our solution and LEACH protocols in MATLAB. In these simulations we considered 100 nodes in a $100 * 100$ meter area. Table 2 represents the simulation results which have been applied in the simulation scenarios.

\begin{tabular}{|l|c|}
\hline \multicolumn{2}{|c|}{ Table 2. Simulation Parameters } \\
\hline Parameter & Value \\
\hline Number of nodes & 100 nodes \\
\hline Network size & $100 * 100$ \\
\hline BS location & $\begin{array}{c}(100,100) \text { and } \\
(50,150)\end{array}$ \\
\hline Data packet size & $4000 \mathrm{bit}$ \\
\hline Initial energy & $\begin{array}{c}0.2 \mathrm{~J} \text { and } \\
0.5 \mathrm{~J}\end{array}$ \\
\hline Aggregation ratio & $10 \%$ \\
\hline $\mathrm{E}_{\text {elect }}$ & $50 \mathrm{nj} / \mathrm{bit}$ \\
\hline$\varepsilon_{\mathrm{fs}}$ & $10 \mathrm{pj} / \mathrm{bit} / \mathrm{m}^{2}$ \\
\hline$\varepsilon_{\text {amp }}$ & $0.0013 \mathrm{pj} / \mathrm{bit} / \mathrm{m}^{4}$ \\
\hline $\mathrm{E}_{\mathrm{DA}}$ & $5 \mathrm{nj} / \mathrm{bit}$ \\
\hline
\end{tabular}

As indicated in Figure 8, our clustering solution creates more symmetric clusters and this reduces the total distance of intra-cluster communication with clusterhead. Also, clusterheads are positioned far from each other to have less common coverage area. 


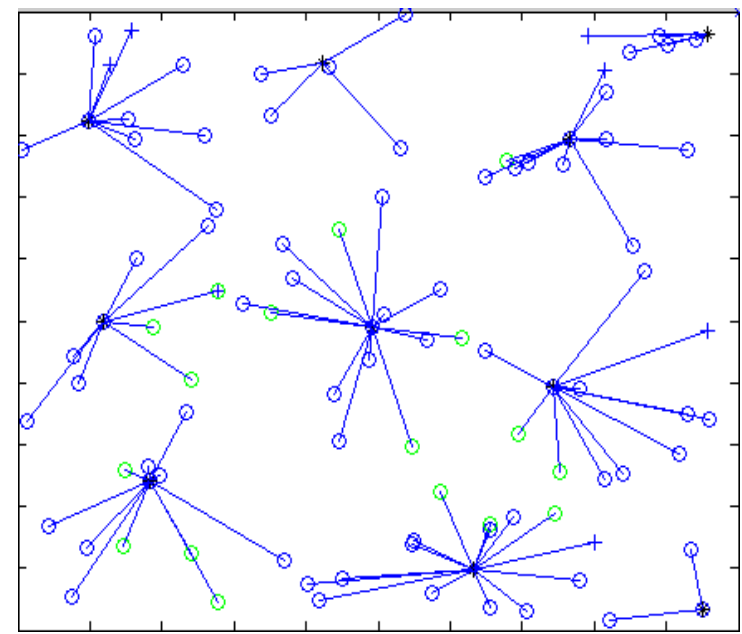

Figure 8. Clustering with Proposed Solution

In our simulations, we have considered three scenarios in which the sink node is positioned in the different locations and two kind of initial energy is used. In the first scenario, the sink node is located at $(100,100)$ location. Also, in this scenario, we have applied 0.2J initial energy in every sensor node. Figure 9 represents the number of dead nodes in each execution round of LEACH and in our proposed solution in the first scenario. As indicated in this figure, our solution improves the first dead node and the total number of nodes until each round. This simulation is conducted at 700 rounds.

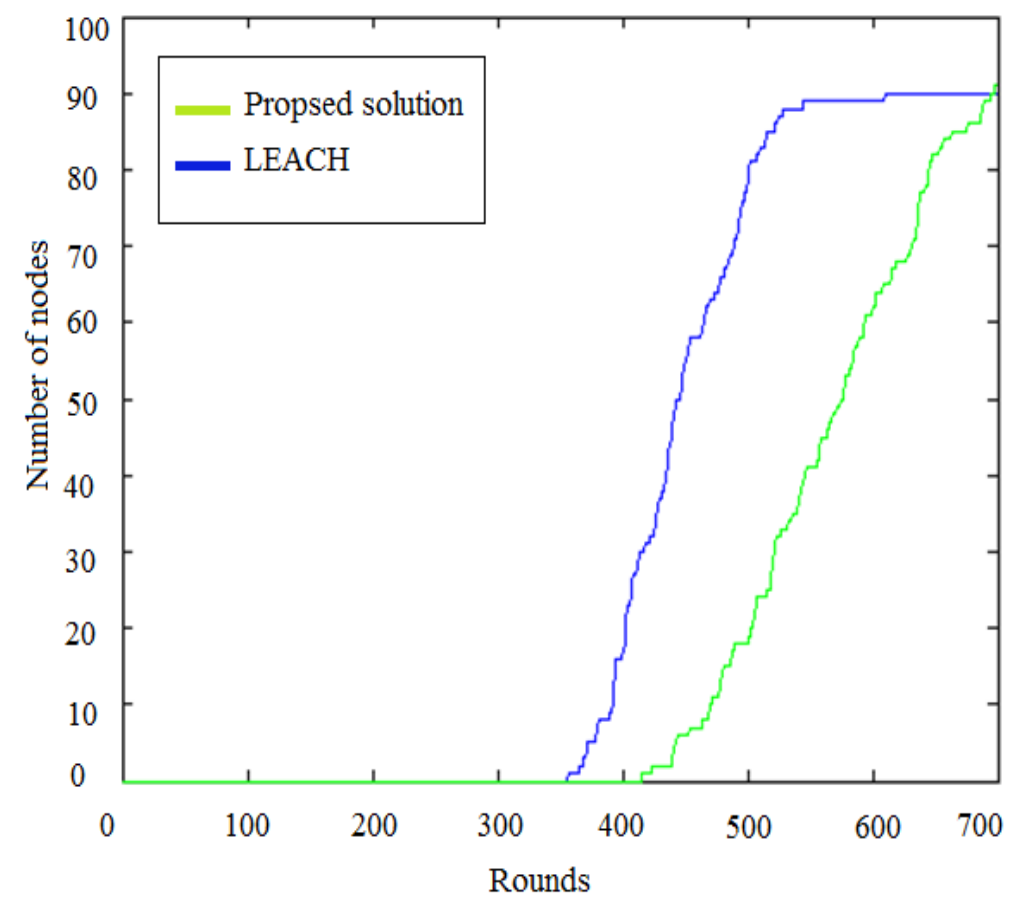

Figure 9. Number of Dead Nodes in First Scenario

In the second scenario we have applied $0.5 \mathrm{~J}$ initial energy in the sensor nodes and the sink node is located at $(100,100)$ location. This simulation is performed at 1000 rounds. 


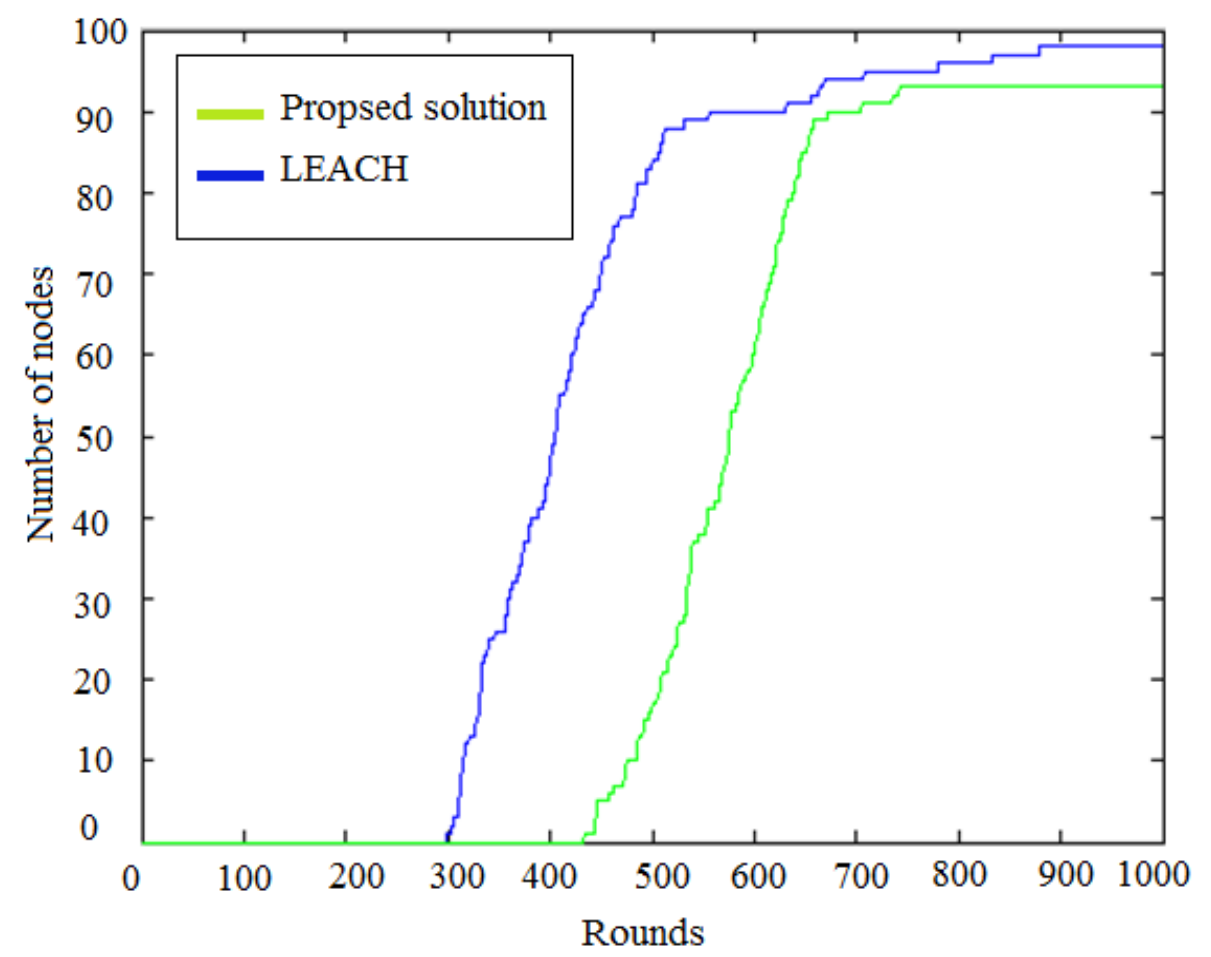

Figure 10. Number of Dead Nodes in Second Scenario

In the third scenario, the sink node is located farther at $(50,150)$ location which is outside of the WSN. As indicated in Figure 10, in this scenario, sensor nodes die sooner, because they clusterheads should transmit their data to a sink node which is located in farther distance. Also, 0.5J initial energy is applied for each sensor node and advanced nodes have double of this value. Moreover, this simulation is conducted at 1000 rounds.

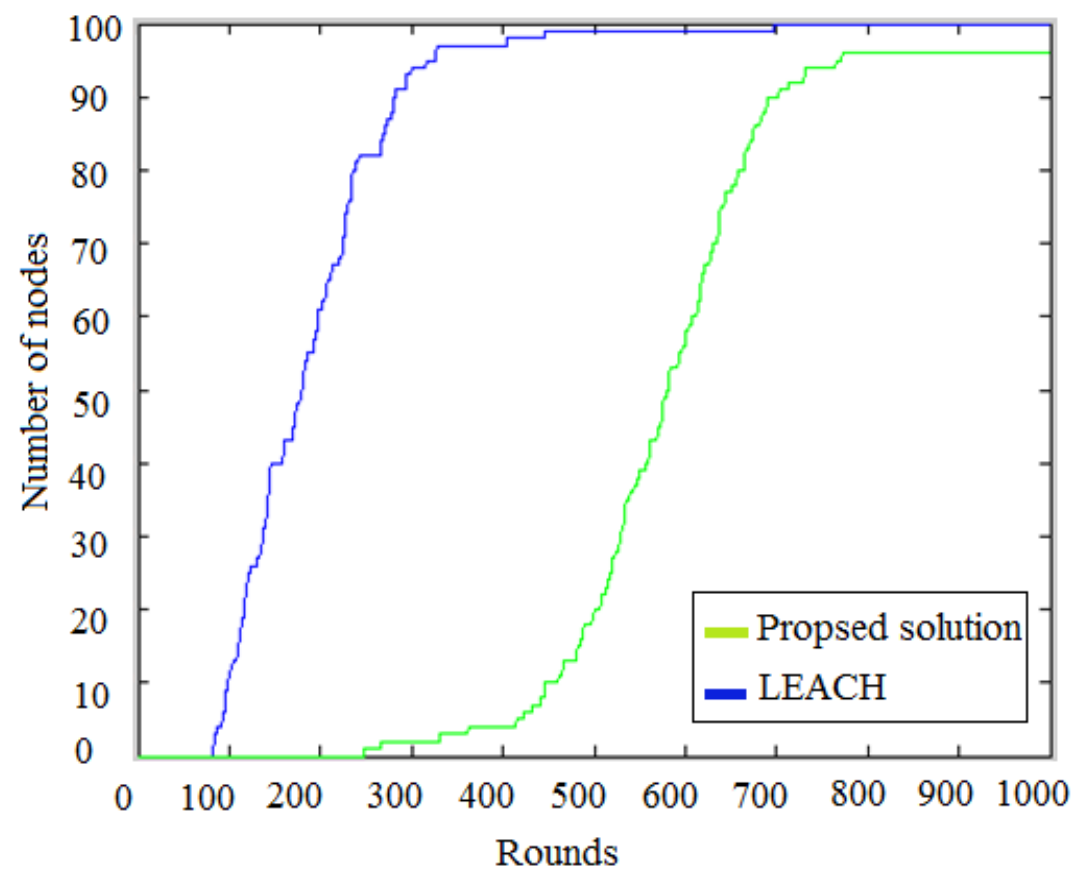

Figure 11. Number of Dead Nodes in the Third Scenario 


\section{Conclusion}

Energy is one of the important issues which should be considered in the WSN. Clustering is a critical network management operation that can be used to reduce the energy consumption of network and improves the lifetime of WSN. In this paper, we presented a fuzzy clustering method which changed the probabilistic clusterhead selection in the LEACH protocol. The fuzzy system used in the inference engine of this scheme is the Mamdani fuzzy system which is a simple rule-base method. This scheme creates symmetric clusters and reduces the total node to clusterhead distance. This finally, reduces the energy consumption of sensors and improves the lifetime of WSN better than LEACH protocol.

\section{References}

[1] V. Katiyar, N. Chand, and S. Soni, "Clustering algorithms for heterogeneous wireless sensor network: A survey," International Journal of Advanced Networking and Applications, vol. 2, pp. 745-754, 2011.

[2] F. Bajaber and I. Awan, "Adaptive decentralized re-clustering protocol for wireless sensor networks," Journal of Computer and System Sciences, vol. 77, pp. 282-292, 2011.

[3] M. Bidaki and M. Masdari, "Reputation-Based Clustering Algorithms in Mobile Ad Hoc Networks," International Journal of Advanced Science \& Technology. May2013, vol. 54, pp. p1-11, 2013.

[4] M. Masdari, S. M. Bazarchi, and M. Bidaki, "Analysis of secure LEACH-based clustering protocols in wireless sensor networks," Journal of Network and Computer Applications, vol. 36, pp. 1243-1260, 2013.

[5] L. Karim, N. Nasser, and T. Sheltami, "A fault-tolerant energy-efficient clustering protocol of a wireless sensor network," Wireless Communications and Mobile Computing, vol. 14, pp. 175-185, 2014.

[6] O. Boyinbode, H. Le, and M. Takizawa, "A survey on clustering algorithms for wireless sensor networks," International Journal of Space-Based and Situated Computing, vol. 1, pp. 130-136, 2011.

[7] M. Masdari and M. Tanabi, "Multipath Routing protocols in Wireless Sensor Networks: A Survey and Analysis," International Journal of future generation communication and networking, vol. 6, pp. 181192, 2013.

[8] W. R. Heinzelman, A. Chandrakasan, and H. Balakrishnan, "Energy-efficient communication protocol for wireless microsensor networks," in System sciences, 2000. Proceedings of the 33rd annual Hawaii international conference on, 2000, p. 10 pp. vol. 2.

[9] Z. Wang, M. Jiang, Y. Hu, and H. Li, "An incremental learning method based on probabilistic neural networks and adjustable fuzzy clustering for human activity recognition by using wearable sensors," Information Technology in Biomedicine, IEEE Transactions on, vol. 16, pp. 691-699, 2012.

[10] M. Bidaki and M. Masdari, "Reputation-Based Clustering Algorithms in Mobile Ad Hoc Networks," International Journal of Advanced Science and Technology, vol. 54, pp. 1-12, 2013.

[11] M. Aslam, N. Javaid, A. Rahim, U. Nazir, A. Bibi, and Z. Khan, "Survey of extended LEACH-Based clustering routing protocols for wireless sensor networks," in High Performance Computing and Communication \& 2012 IEEE 9th International Conference on Embedded Software and Systems (HPCC-ICESS), 2012 IEEE 14th International Conference on, 2012, pp. 1232-1238.

[12] Y.-F. Huang, W.-H. Luo, J. Sum, L.-H. Chang, C.-W. Chang, and R.-C. Chen, "Lifetime Performance of an energy efficient clustering algorithm for cluster-based wireless sensor networks," in Frontiers of High Performance Computing and Networking ISPA 2007 Workshops, 2007, pp. 455-464.

[13] D. Kumar, T. C. Aseri, and R. Patel, "EEHC: Energy efficient heterogeneous clustered scheme for wireless sensor networks," Computer Communications, vol. 32, pp. 662-667, 2009.

[14] M. A. AbdulAlim, Y. C. Wu, and W. Wang, "A fuzzy based clustering protocol for energy-efficient wireless sensor networks," in Advanced Materials Research, 2013, pp. 685-690.

[15] L. Barolli, "A speed-aware handover system for wireless cellular networks based on fuzzy logic," Mobile Information Systems, vol. 4, pp. 1-12, 2008.

[16] O. Younis and S. Fahmy, "HEED: a hybrid, energy-efficient, distributed clustering approach for ad hoc sensor networks," Mobile Computing, IEEE Transactions on, vol. 3, pp. 366-379, 2004.

[17] Z. Yu, X. Fu, Y. Cai, and M. C. Vuran, "A reliable energy-efficient multi-level routing algorithm for wireless sensor networks using Fuzzy Petri Nets," Sensors, vol. 11, pp. 3381-3400, 2011. 